\title{
Digital technologies and musical participation for people with
} intellectual disabilities

\author{
Barbara Adkins ${ }^{\mathrm{a}}$, Jennifer Summerville ${ }^{\mathrm{b}}$, \\ Marie Knox ${ }^{c}$, Andrew Brown ${ }^{\mathrm{d}}$, \\ and Steven Dillon ${ }^{\mathrm{e}}$ \\ ${ }^{a}$ Creative Industries Faculty, Queensland University of Technology, Brisbane, \\ Queensland, Australia \\ "b.adkins@qut.edu.au" \\ ${ }^{\mathrm{b}}$ Health and Community Services Workforce Council, Brisbane, Queensland, \\ Australia \\ “jenny.summerville@gmail.com" \\ ${ }^{c}$ School of Social Work and Human Services, Queensland University of Technology, \\ Brisbane, Queensland, Australia \\ "m.knox@qut.edu.au" \\ ${ }^{\mathrm{d}}$ Queensland Conservatorium of Music, Griffith University, Brisbane, Queensland, \\ Australia \\ "andrew.r.brown@griffith.edu.au" \\ ${ }^{\mathrm{e}}$ Creative Industries Faculty, Queensland University of Technology, Brisbane, \\ Queensland, Australia
}

Barbara Adkins is a sociologist whose research focuses on the role of design in social and cultural life. She is an associate professor in the School of Design in the Creative Industries Faculty at the Queensland University of Technology in Brisbane, Australia and was formerly education manager and researcher at the Australasian Cooperative Research Centre for Interaction Design.

Jennifer Summerville is a sociologist specialising in participatory research and evaluation methods. She is currently the Research and Development Coordinator at the Health and Community Services Workforce Council and a Visiting Senior Research Fellow at the Queensland University of Technology. Jenny is a keen advocate for building research capacity as a foundation for inclusion and continuous improvement. Her research interests centre on strategies and applications to support workforce planning and development in the health and community services industries.

Marie Knox has an extensive background in disability studies research. She is currently a Visiting Fellow in the School of Public Health and Social Work at the Queensland University of Technology, Brisbane, and a Senior Research Fellow at the Centre for Disability Studies affiliated with the University of Sydney where she leads the research program.

Andrew Brown Andrew R. Brown is a computational artist, researcher and educator. $\mathrm{He}$ is Professor of Digital Arts at the Queensland Conservatorium of Music, Griffith University, in Australia and was previously the Research Manager for the Australasian CRC for Interaction Design (ACID). Andrew is an active computational artist working in music and visual domains. His research interests include technology 
support for creativity and learning, computational aesthetics and the philosophy of technology.

Steve C. Dillon Sadly, our colleague and friend, Dr Steve Dillon, passed away on April $1^{\text {st }}$ 2012. He was a singer songwriter, music educator and musicologist, and a senior lecturer in Music and Sound at Queensland University of Technology. He was director of save to DISC Research Network and project Leader of the Network Jamming Research Group. His research interests were meaningful engagement with music making and designing digital media technologies and relational pedagogies to provide access to cognitive growth, health and well being through music making. He leaves behind an important legacy in researching and developing tools to support participation in music making. 
Adkins, Barbara, Marie Knox, Steve Dillon, Andrew R. Brown, and Jennifer Summerville. "Digital Technologies and Musical Participation for People with Intellectual Disabilities." New Media and Society 14, no. 7 (2012).

http://nms.sagepub.com/content/early/2012/09/24/1461444812457338.full.

\title{
Digital technologies and musical participation for people with
} intellectual disabilities

\author{
Abstract \\ Research on the aspirations of people with intellectual disabilities documents \\ the importance of alternative zones of inclusion where they can assert their \\ own definitions of ability and normality. This stands in contrast to assumptions \\ concerning technology and disability that position technology as 'normalising' \\ the disabled body. This paper reports on the role of a digital music jamming \\ tool in providing access to creative practice by people with intellectual \\ disabilities. The tool contributed to the development of a spatio-temporal zone \\ to enable aesthetic agency within and beyond the contexts of \\ deinstitutionalised care. The research identifies the interactions among tools, \\ individuals and groups that facilitated participants' agency in shaping the form \\ of musical practice. Further, we document the properties of emergent \\ interaction - supported by a tool oriented to enabling music improvisation - as \\ potentially resisting assumptions regarding normalisation.
}

\section{Keywords}

Digital technologies, intellectual disability, music improvisation, cultural participation 
Adkins, Barbara, Marie Knox, Steve Dillon, Andrew R. Brown, and Jennifer Summerville. "Digital Technologies and Musical Participation for People with Intellectual Disabilities." New Media and Society 14, no. 7 (2012).

http://nms.sagepub.com/content/early/2012/09/24/1461444812457338.full. 
Adkins, Barbara, Marie Knox, Steve Dillon, Andrew R. Brown, and Jennifer Summerville. "Digital Technologies and Musical Participation for People with Intellectual Disabilities." New Media and Society 14, no. 7 (2012).

\section{Introduction}

This paper conceptualises and empirically investigates the role of a digital 'music jamming' tool in promoting musical participation for people with intellectual disabilities. The conceptualisation of participation responds to a call for caution regarding assumptions about the role of technologies with respect to disability, particularly the implication that technologies are oriented to 'normalising' the disabled body (Moser, 2006). The analysis problematises such assumptions first by identifying the specific relationships and practices at stake in participation in the field of culture (Bourdieu, 1984; Warde, 2004), and then locates questions regarding the role of digital technology in this context. Second, the paper conceptualises key elements relevant to the nature of practices enabled by a particular digital music jamming tool and implications for the development and application of aesthetic agency, including relationships of recognition (Ricoeur, 2005). Third, we present an analysis of interview and observational data collected from participants at two day centres, for people with intellectual disabilities, prior to and following their three-week use of the digital jamming tool. The research identifies the participants' orientations to music and technology, and the relationships of recognition evolving throughout the course of their use of the tool.

The analysis reveals properties of the music experience in terms of emergence and improvisation that both enhance opportunities for aesthetic engagement and provide the potential to resist assumptions regarding the normalising function of technology. Improvisation is a key feature of group creativity, and, in the context of music, refers to the activity of 
Adkins, Barbara, Marie Knox, Steve Dillon, Andrew R. Brown, and Jennifer Summerville. "Digital Technologies and Musical Participation for People with Intellectual Disabilities." New Media and Society 14, no. 7 (2012).

http://nms.sagepub.com/content/early/2012/09/24/1461444812457338.full.

extemporaneous 'in the moment' music making (Hickey, 2009: 287), in which musicians' choices of pre-existing musical structures or motifs are adapted in the context of the music making process (Sawyer, 2006). In the case of group creativity, the improvisations of players together have an emergent property where the outcome is contingent and represents a 'whole which is greater than the sum of its parts' (Sawyer, 2006: 148). Thus, supporting group improvisation generates a setting in which the musical sense legitimately unfolds, and, as such, at any point in time, judgements of individual contributions, correct or incorrect, appropriate or inappropriate practice are not consistent with the nature of the activity. This reflects the nature of learning involved in improvisation, which is argued to require the development of improvisational dispositions rather than a focus on skills alone (Hickey, 2009). In this context, digital music improvisation tools can provide opportunities for participants to develop and assert their own definitions of normality and ability in and through collective aesthetic agency.

\section{Disability and the 'promise of technology'}

A recent review of the 'promises of technology' for people with disabilities identifies the need for a critical examination of assumptions underpinning this 'promise'. In particular, the review proposes that some accounts of the links between technologies and disability are founded on assumptions regarding normality and normalisation that do not serve people with disabilities. Moser (2006) suggests:

the mobilization of new technologies works to build an order of the normal and turn disabled people into competent normal subjects. 
Adkins, Barbara, Marie Knox, Steve Dillon, Andrew R. Brown, and Jennifer Summerville. "Digital Technologies and Musical Participation for People with Intellectual Disabilities." New Media and Society 14, no. 7 (2012).

http://nms.sagepub.com/content/early/2012/09/24/1461444812457338.full.

However, this strategy based on compensation achieves its goals only at a very high price: by continuing to reproduce boundaries between abled and disabled, and normal and deviant, which constitute some people as disabled in the first place. There are thus limits to normalization. And so, notwithstanding their generative and transformative power, technologies working within an order of the normal are implicated in the (re)production of the asymmetries that they and it seek to undo (Moser, 2006: 373). The implications of this observation inform the present study. The relationships through which technologies are seen to 'assist or 'support the participation of people with intellectual disabilities need to be scrutinised for their capacity to provide experiences that withstand assumptions regarding normalisation. In particular, it is crucial to provide for creative practice that embraces difference and, in this context, to provide opportunities for participation in the field of culture (Bourdieu, 1984) on the terms of those with intellectual disabilities rather than abstract norms of ability and disability (Moser, 2000). Bourdieu's use of the term, 'field', addresses the tendency for broader social inequities to take specific forms in particular social domains. In this respect, fields such as culture, education, economic life may exhibit their own structure of positions and principles of inclusion and participation (Bourdieu, 1984).

In the context of the stakes and processes of participation in the field of culture, as in other social fields, people with intellectual disabilities experience a double disadvantage. Intellectual disability is both a social construction, as well as an ontological reality (Klotz, 2004). People with this disability can experience difficulties arising from their impairment, 'characterized by 
Adkins, Barbara, Marie Knox, Steve Dillon, Andrew R. Brown, and Jennifer Summerville. "Digital Technologies and Musical Participation for People with Intellectual Disabilities." New Media and Society 14, no. 7 (2012).

http://nms.sagepub.com/content/early/2012/09/24/1461444812457338.full.

significant limitations both in intellectual functioning and in adaptive behavior as expressed in conceptual, social, and practical adaptive skills' (Schalock, 2010: 1). Further, they may equally suffer from society's response to that impairment, which, in itself, leads to limited participation opportunities.

Studies focusing on the relationship between information and communication technologies and participation by people with intellectual disabilities in social fields are cautiously optimistic about the potential for digital and web based applications to support participation. In their review of technology use by students with intellectual disabilities, Hendricks and Wehman (2009) point to beneficial use of information and communication technologies in areas such as community integration, education, and recreation and leisure, suggesting an increased focus on cognitive accessibility of the technology to enhance participation. Focusing on video telephony, Renblad (2000) documents its capacity to strengthen and broaden social networks, and McClimens and Gordon (2009) provide preliminary evidence of the social value of blogging for people with intellectual disabilities.

These findings raise questions of the relationships underpinning access to-and participation in—social fields and the role of digital technologies in this process. Nind and Seale (2009) point to the importance of conceptual frameworks that emphasise the meanings and dimensions of access relationships as they are understood and prioritised by people with intellectual disabilities. This is proposed as a crucial measure to avoid reductionism in policy and program approaches and, more positively, to ensure that these are grounded in the preferences and practices of this group. Building on these studies in light of the questions posed by Moser (2006), it is 
Adkins, Barbara, Marie Knox, Steve Dillon, Andrew R. Brown, and Jennifer Summerville. "Digital Technologies and Musical Participation for People with Intellectual Disabilities." New Media and Society 14, no. 7 (2012).

http://nms.sagepub.com/content/early/2012/09/24/1461444812457338.full.

important to establish a framework for understanding and addressing relationships with digital technologies that emphasise their priorities. The purpose of this research is to intervene in assumptions pertaining to the normalising function of technology, by replacing them with a framework that sensitises inquiry to the opportunities for aesthetic engagement on the terms of people with intellectual disabilities.

\section{Intellectual disability: conceptualising participation}

In recent years the discipline of geography has made an important contribution to the conceptualisation of participation of people with intellectual disabilities. In their review of this area, Philo and Metzel (2005) identify key social and cultural questions that attend the relationships of segregation and deinstitutionalisation that continue to affect the lives of people with intellectual disabilities. In this work, it is broadly argued that while deinstitutionalisation paves the way for the location of people with intellectual disabilities in community settings, it cannot be assumed that this addresses the social exclusion of this group. The ongoing relevance of problematising the nature of inclusion and participation is documented in research by Metzel which points to the way patterns of dependency that characterised institutionalisation can be inadvertently recreated in programs in community settings (Metzel, 2005). In this context, research is exploring alternative possibilities whereby (deinstitutionalised) intellectually disabled people might be welcomed into the life of the community on terms more relevant to them than to the assumptions of a neglectful mainstream (Hall and Kearns, 2001; Philo and Metzel, 2005). In line with these principles, Walker advocates a focus on the nature of 
Adkins, Barbara, Marie Knox, Steve Dillon, Andrew R. Brown, and Jennifer Summerville. "Digital Technologies and Musical Participation for People with Intellectual Disabilities." New Media and Society 14, no. 7 (2012).

http://nms.sagepub.com/content/early/2012/09/24/1461444812457338.full.

'community presence that works for this group, promoting a feeling of belonging connection and companionship (Walker, 1999). Hall's research on social geographies of learning disability ${ }^{1}$ describes the way people with a learning disability place value and emphasis on alternative spaces of inclusion. Hall proposes a research focus prioritising places, networks and activities where 'new forms of "normality" and inclusion can be shaped' (Hall, 2004).

From a sociological perspective, Schelly (2008) provides an in-depth examination of the relationships that might promote choice rather than dependency for people with intellectual disability, arguing that community based supports and services need to be sensitised to difference. Similarly, Rubin et al (2001) emphasise the capacity of people with intellectual disabilities to assert their versions of ability and thus influence the ordering of the contexts in which they participate. Their research identifies the need for a concept of participation that includes 'having a stake and effect in redefining the notion of ability' (Rubin et al, 2001: 426).

Given these conceptual principles regarding notions of participation for people with intellectual disabilities, what are the implications for their use of digital technologies oriented to enhancing participation in arts activities?

\section{Digital technology as a participatory tool: The case of}

\section{jam2jam}

\footnotetext{
1 "Learning Disability is often used in the UK context in preference to the term "Intellectual Disability"
} 
Adkins, Barbara, Marie Knox, Steve Dillon, Andrew R. Brown, and Jennifer Summerville. "Digital Technologies and Musical Participation for People with Intellectual Disabilities." New Media and Society 14, no. 7 (2012).

http://nms.sagepub.com/content/early/2012/09/24/1461444812457338.full.

Our examination of the relationship between digital technology and participation for people with intellectual disabilities occurs in the context of their use of a digital music jamming tool. In terms of recent recommendations in arts sociology, the use of the tool in everyday life provides an opportunity to investigate the effects of digitally mediated music as it is mobilised in action (De Nora, 2003; De la Fuentes, 2007) and, for our purposes, the possibilities it provides to resist normalisation assumptions. As such, in place of implicit assumptions concerning normalisation, the use of the tool is studied as a site of practices that have implications for participation (Warde, 2004) in the field of culture on the terms of people with intellectual disabilities.

The application of this tool specifically to promote participation for people with intellectual disabilities is supported by a body of research on the benefits of music improvisation processes for people with a range of disabilities (Wigram et al, 2011; Hooper et al, 2008; Stephenson, 2006; Duffy, 2000). These include the provision of opportunities for spontaneity, expression and forms of feeling beyond the specific meanings of words, and the communicative constraints of physical and cognitive disabilities. Collaborative improvisation is argued to enable the representation of the group and the acknowledgement of the roles of individuals as they emerge in the music (Skewes and Wigram, 2002). Further, benefits of these improvisation processes are found specifically in the context of people with intellectual disabilities, when compared to more structured music activities (Edgerton, 1994).

Jam2jam is a tool based on generative principles and thus underpinned by an algorithmic music system which itself exerts at least partial control in the 
Adkins, Barbara, Marie Knox, Steve Dillon, Andrew R. Brown, and Jennifer Summerville. "Digital Technologies and Musical Participation for People with Intellectual Disabilities." New Media and Society 14, no. 7 (2012).

http://nms.sagepub.com/content/early/2012/09/24/1461444812457338.full.

development of a worSpecifically, the generative system scaffolds music making and thus provides access to collaborative interaction while still allowing for the development of expertise and expression. These systems can be developed to enable technically 'inexpert' participants to develop worksin this case, music performances. The jam2jam system used in this study was controlled via a set of MIDI (Musical Instrument Digital Interface) - based slider interfaces where each interface corresponded to a synthesized instrument and each fader on the interface to an algorithmically mediated musical parameter such as dynamic range, pitch range, density, timbral brightness, part volume, and so on. (Dillon, 2006; Brown et al, 2009).

Figure 1 Here

Figure 1 The jam2jam AV Interface showing graphic symbols for parameters and instruments and MIDI-based slider controllers.

Extended explanations, photos and videos of jam2jam systems can be found at: http://explodingart.com/jam2jam/jam2jam/About.html .

This raises the question of the properties of the digital tool relevant to musical participation that enables people to assert their own definitions of ability and normalityThe jam2jam tool was developed to support creative collaboration across a wide variety of groups including school children, and young people from diverse sociodemographic backgrounds. A central property of the jam2jam tool is its provision of a variety of given musical styles 
Adkins, Barbara, Marie Knox, Steve Dillon, Andrew R. Brown, and Jennifer Summerville. "Digital Technologies and Musical Participation for People with Intellectual Disabilities." New Media and Society 14, no. 7 (2012).

http://nms.sagepub.com/content/early/2012/09/24/1461444812457338.full.

enabled by a series of generative musical rules, based on the formulae and logic of emergent musical forms such as rock and pop. Within these styles, participants can vary a range of parameters such as timbre, tempo, density, volume, and adjust these for specific instruments during a performance. This process allows the user to generate a musical output, which is in line with the particular style chosen and without needing the traditionally required performance skills, knowledge and techniques associated with the rules of different instrumental practices and musical styles.

Jam2jam ensures that all musical notes and phrases follow harmonic principles, ensuring that the output will possess the full instrumental and harmonic characteristics of the chosen style. The user acts as an arranger and conductor of the musical output through a series of interface control mechanisms. In addition to the capacity of the tool to accommodate diverse styles, instrument combinations and sound qualities, there is also a facility for video display with opportunities to capture the images of performers and apply specific colour and pattern affects, and the audio-visual recording and replay of performances. The tool enables individual or collective improvisation while ensuring a performance that is based on given harmonic, rhythmic and instrumental principles. This kind of environment has similarities to jazz improvisation around a composed harmonic structure and melodic 'head' that provides a vehicle for broad expressive variation in timbre and rhythm as well as, dynamic and textural aspects of the music. Composer, John Zorn, describes this kind of improvisation as 'game pieces: 'complex systems harnessing improvisers in flexible compositional formats' (Zorn, 2004). 
Adkins, Barbara, Marie Knox, Steve Dillon, Andrew R. Brown, and Jennifer Summerville. "Digital Technologies and Musical Participation for People with Intellectual Disabilities." New Media and Society 14, no. 7 (2012).

http://nms.sagepub.com/content/early/2012/09/24/1461444812457338.full.

The tool constitutes an improvisational environment in the sense that activities with it involve neither performance of a notated score nor composition from first principles. The sound and visual materials provided in the system can be manipulated in real-time and the musical form unfolds from this manipulation. This kind of improvisation is appropriate for the goals of participation because it allows a playful interaction that is neither highly prescribed nor intimidatingly open.

Thus jam2jam provides the resources for 'jamming', that is, loosely structured musical performances. While clearly the digital tool provides supports to improvisation processes, such as constraints around harmonic, rhythmic possibilities, that are absent in traditional jamming contexts, some crucial features of jamming are retained: It affords players the opportunity to develop musical ideas through the observation and imitation of others (Scott, 2004), and prioritises the 'happy science of the moment' (Marmande and Johnson, 1996). Further, as Sawyer points out, some form of given structure is a central feature of improvisation processes in jamming in that participants 'draw on culturally shared emblems and stereotypes, which in some sense are pre-existing structures' (Sawyer, 2000: 184). In these contexts the music is emergent in that it comes from the interactions in the group (Sawyer, $2000: 182)$, meaning that the outcome is contingent and not reducible to the aggregate contributions of participants (Sawyer, 2006). The digitallysupported form of jamming retains these characteristics, with algorithms providing an array of pre-existing musical structures while the collaborative management of musical parameters provide for its emergent properties. 
Adkins, Barbara, Marie Knox, Steve Dillon, Andrew R. Brown, and Jennifer Summerville. "Digital Technologies and Musical Participation for People with Intellectual Disabilities." New Media and Society 14, no. 7 (2012).

http://nms.sagepub.com/content/early/2012/09/24/1461444812457338.full.

These particular properties of digitally supported music jamming raise the question of a framework for understanding the specific dimensions and processes of musical practices in this context, which sit at the intersection between the fields of culture and information and communication technologies. We applied Paul Ricoeur's incisive analysis of the 'course of recognition'-a key requirement for participation—-that is sensitised to context and temporality and thus suited to capturing 'emergence' and the meanings and understandings of participants. Ricoeur breaks up the course of recognition into three elements: 'recognition as identification ', 'recognising oneself and 'mutual recognition' (Ricoeur, 2005). The purpose of these three components is to understand recognition analytically in terms of these discrete elements and to see them as potentially unfolding in a course or sequence.

For Ricoeur, 'recognition as identification' refers to the process of identifying or distinguishing something or someone, thus illuminating a key precursor to participation in understanding the nature and meaning of a context such that practices can be attuned to it. The next element, 'recognising oneself', involves understanding the difference between self and other, assigning actions to ourselves and others, and taking responsibility for them. This process clearly aligns with the requirements for agency in participation. The third element, 'mutual recognition', refers to the recognition of oneself by others, and is another key condition under which we develop and enact a sense of agency, of being understood, and experience self confidence, self respect and social esteem. 
Adkins, Barbara, Marie Knox, Steve Dillon, Andrew R. Brown, and Jennifer Summerville. "Digital Technologies and Musical Participation for People with Intellectual Disabilities." New Media and Society 14, no. 7 (2012).

http://nms.sagepub.com/content/early/2012/09/24/1461444812457338.full.

This conceptual framework is very suited to understanding the variety of relationships and networks involved in musical practice in the context of using digital tools; it allows for both human and non-human agency in the evolution of recognition in each of these elements. In our study, this conceptualisation of recognition is used to sensitise the analysis to the configuration of relationships involved in the enactment of aesthetic agency. Specifically, it enables us to raise questions concerning the recognition of a context as offering possibilities of aesthetic practice, of oneself and others as having the potential to contribute to this practice individually and/or collaboratively, and of musical works as legitimately attracting recognition from others. Furthermore, as a framework for capturing these details of agency it is specifically suited to our concern to examine the role of the digital tool in participants' adoption of their own notions of normality and musical practice, and thus resist prevalent assumptions regarding technology and normalisation.

\section{Design of the study}

We investigated the participatory possibilities of the jam2jam tool for people with intellectual disabilities based on the theoretical and conceptual considerations outlined above. The research took place in two day centres in Brisbane, Australia, for adults with significant intellectual disabilities. Australia, like most other developed countries, has seen changes in services to people with intellectual disabilities in recognition of their rights to have access to - and participate in - community. The day centres, as part of this move, aim to support people with more severe intellectual disabilities (those considered 
Adkins, Barbara, Marie Knox, Steve Dillon, Andrew R. Brown, and Jennifer Summerville. "Digital Technologies and Musical Participation for People with Intellectual Disabilities." New Media and Society 14, no. 7 (2012).

http://nms.sagepub.com/content/early/2012/09/24/1461444812457338.full.

unable to be employed in competitive or supported employment). They aim to foster the social inclusion of the people accessing these services. However, while community living has greatly enhanced the quality of the lives of people with intellectual disabilities, social inclusion remains a somewhat elusive ideal. Institutional discourses continue to pervade the lives of many such people (Hamlin and Oakes, 2008; Mansell, 2006) and they still lead relatively segregated lives. This is particularly so for people with severe intellectual disabilities--the people participating in the day centres. The use of technology has been recognised as one possible means of addressing this issue (Goggin and Newell, 2003), and the programs operating at the two centres involved in this study use some information and communication technology activities involving internet access and games played on platforms such as Xbox and Playstation Hence, the service users have some familiarity with the use of digital tools. However, this raises the question of the role of this technology in enabling participation in various fields, and, in particular, the possibilities for participation offered by a specific kind of digital tool oriented to supporting improvisation.

The research was conducted in three stages: 1) interviews with the service users of the day centres to identify their experience of cultural participation and information and communication technology prior to exposure to the jam2jam tool; 2) observations of their interaction with the tool over a period of three weeks at each of two day centres; and 3) group interviews to elicit their descriptions of their use of the tool following the three week period. The interviews sought to understand participants' description of the current contexts in which they participate in music and technology activities, and, 
Adkins, Barbara, Marie Knox, Steve Dillon, Andrew R. Brown, and Jennifer Summerville. "Digital Technologies and Musical Participation for People with Intellectual Disabilities." New Media and Society 14, no. 7 (2012).

http://nms.sagepub.com/content/early/2012/09/24/1461444812457338.full.

following the experience with jam2jam, their assessments of working with the digital tool. The observational study was conducted in the day centres and aimed to capture and analyse — through video analysis_- the in situ relationships to information and communication technology and cultural participation experienced by the service users. Used over several weeks, these videos helped identify the nature of recognition relationships involved in aesthetic participation as they emerged in the use of a tool designed to support improvisation.

\section{Descriptions of participation in music and information and communication technologies by people with intellectual}

\section{disabilities}

From the interviews conducted prior to and following their use of jam2jam, participants' descriptions indicated the operation of recognition relationships in their daily practices. In terms of 'recognition as identification', they enjoyed the opportunity to display their familiarity with elements of music and ICT:

- Knowledge of music styles, songs, artists and bands, and favourite instruments used in the music program at the centres such as cymbals, tambourines, shakers and drums; and

- The nature and processes involved in engaging in specific computer based applications, such as games, and internet searching, using terms such as Playstation, Xbox, and Google.

Superimposed on this level of connection with these fields was the clear capacity and interest in asserting agency ('recognising oneself') within these 
Adkins, Barbara, Marie Knox, Steve Dillon, Andrew R. Brown, and Jennifer Summerville. "Digital Technologies and Musical Participation for People with Intellectual Disabilities." New Media and Society 14, no. 7 (2012).

http://nms.sagepub.com/content/early/2012/09/24/1461444812457338.full.

domains. In describing participation in music and dance programs at the centres, participants described preferences for playing particular instruments in music activities, singing and dancing and conveying a sense of fun in the interaction generated in the sessions. For example, a participant, Greg, described the music activity at his centre as 'We do like this [clapping hands]', and his favourite musical instruments: 'Ah cymbal, I mean like that [mimicking using a tambourine with hand movements]'. When asked why he liked the tambourine the best he said: 'because it's my favourite. Special'. Many participants described specific tastes and preferences in music experienced at home. For example, Louise talked about listening to CDs in her lounge room, liking Rolf Harris (singing 'Jake the Peg') and Abba, playing the piano and dancing. She also enjoyed dancing at the centre. These responses suggested the need to consider possibilities for combining acoustic instrument performance with digital tools in future design concepts.

In relation to information and communication technologies, agency was asserted in preferences for specific games and web-based platforms attesting to experience with, and use of, these applications. Several participants described using the computer at home. For example, Louise spoke of 'writing a lot of things' on the computer, including her own stories, and playing solitaire. Greg stated a preference for using 'Google', which he used to search for the towns where family members resided. Another participant also demonstrated the process of changing the AV from his stereo (which he used for CDs and records) to the computer in order to watch DVDs in his bedroom.

Such practices were given more emphasis in the group interviews following the use of jam2jam, where interviewees elaborated on their current 
Adkins, Barbara, Marie Knox, Steve Dillon, Andrew R. Brown, and Jennifer Summerville. "Digital Technologies and Musical Participation for People with Intellectual Disabilities." New Media and Society 14, no. 7 (2012).

http://nms.sagepub.com/content/early/2012/09/24/1461444812457338.full.

use of digital technologies both in terms of broad recreational activities and to access and make music. Regarding recreation and games, participants described playing computer games at their centres. Several described playing ten pin bowling using Nintendo Sports, as well as other games. In general, participants who used computers and game tools eagerly described and demonstrated (when the opportunity arose) their competencies using digital tools. Many elaborated on their skills in particular games (e.g., scores, who they could beat) and/or their abilities to use a computer, including activities of 'downloading files'. Some demonstrated how they could play a computer game, turn on a computer, open an application and play a DVD.

There was also enthusiastic discussion of the use of digital technologies to access and experience music. One participant described her brother downloading music to the MP3 player he gave her: 'you can download it if you have a - l've got my brother's MP3 player. He downloaded me some files last night on it so I get it from his MP3 player and he loads it on for me'. Another spoke about 'making' music by downloading a selection of songs to make his own CD: 'I make music home put shell in computer. Sound music... I make CD... Make Jimmy Barnes, John Farnham... together... I make song.' Overall, digital tools appeared to play a key role in many participants' day-to-day lives; however, the nature of participation with digital tools was typically underpinned by individual engagement with them. That is, the engagement comprised a relationship between an individual and the tool and rarely involved collaboration with others, except in the form of a 'support' relationship where key workers or family members assist them in using the tools. Similarly, a significant finding from participant interviews regarding their 
Adkins, Barbara, Marie Knox, Steve Dillon, Andrew R. Brown, and Jennifer Summerville. "Digital Technologies and Musical Participation for People with Intellectual Disabilities." New Media and Society 14, no. 7 (2012).

http://nms.sagepub.com/content/early/2012/09/24/1461444812457338.full.

current participation in the field of music was that experiences are typically either individual or facilitated in a formal or programmed sense. In this respect, while participants' descriptions emphasised 'recognition as identification' and 'recognising oneself' in the use of digital tools for recreation and music, experiences of mutual recognition-crucial for a sense of the reception and value of creative work-were not volunteered.

This review of participants' descriptions of their experiences with music and information and communication technologies raised the question of the potentially distinctive qualities of the jam2jam tool for the participants regarding its capacity to promote music practice through the full 'course of recognition'. Specifically, observations sought to investigate the recognition experiences enabled by a tool designed to promote improvisation and collaboration in music participation.

\section{Experience with jam2jam: recognition and participation in}

\section{digitally supported emergent music performance}

In both centres jam2jam activities involved the use of a desktop computer and five fader-based control surfaces. The changes made to the faders were represented on a visual interface through moving instrument icons; for example, the control of drums produces movement in the drums icon on the screen. Jam2jam afforded each participant control over six parameters of the production, including volume, density, timbre, pitch range and tempo. In addition to the separate parameter controls for each instrument, there was also an additional control surface to manipulate a video image projected on screen that could be altered through special effects to change colours and 
Adkins, Barbara, Marie Knox, Steve Dillon, Andrew R. Brown, and Jennifer Summerville. "Digital Technologies and Musical Participation for People with Intellectual Disabilities." New Media and Society 14, no. 7 (2012).

http://nms.sagepub.com/content/early/2012/09/24/1461444812457338.full.

produce kaleidoscope effects. The video image could include web camera footage of the performers.

In applying Ricoeur's three part concept of recognition it became clear that as newcomers to collaborative, emergent music participation, participants devoted a lot of their activity to the first component of this - 'recognition as identification' - applied to key aspects of the setting: objects, people and the music context. For example, experimenting with the controllers initially involved moving the sliders up and down quickly and then slowly which helped to determine differences that specific sliders could make to the sound. Further, some participants, having reached a level of understanding of the contribution of their own controllers, sought to assist or prompt co-participants to move their sliders in certain ways, thereby distinguishing between the discrete contributions of, for example, the drum controls and those of the guitar.

Due to the shared screen and web-cam video component of the music activity, participants could also identify themselves on screen by pointing, waving and clapping when they saw their images, and manipulating special effects around them. At times, identification involved symbolic displays of understanding and knowledge associated with the instruments and the activity. For example, one participant after experimenting with the drum controller, started to make drumming gestures to the person next to her while continuing her focus on the music. In the context of musical practice with implications for participation in the field of culture, the experimentation involved awareness and feel for the ways in which the form and style of the performance could be altered through manipulation of controls. 
Adkins, Barbara, Marie Knox, Steve Dillon, Andrew R. Brown, and Jennifer Summerville. "Digital Technologies and Musical Participation for People with Intellectual Disabilities." New Media and Society 14, no. 7 (2012).

http://nms.sagepub.com/content/early/2012/09/24/1461444812457338.full.

The application of the second component of Ricoeur's concept of recognition - 'recognising oneself'--provided crucial insights into the specific properties and value of emergent forms of music making for people with intellectual disabilities. This quality of the activity enabled movement in and out of participation according to individuals' sense of themselves as having roles in the activity at specific points in time. The recognition of the self in the music followed a progression from the involvement of the body to a more specific commitment of the self. This process sometimes functioned to enable participants to move into involvement in the music following their engagement in another activity in the same room. For example, while some participants were involved in a jam2jam performance, another participant was engaged in exercises at the other end of the room. After a while she started conducting her exercises to the time of the music, and slowly moved towards the group as she realised that she was visible on the screen, creating a subtle dance performance. Another participant was noted by a staff member as uncharacteristically pulling her towards the jam2jam activity so that her image could be captured on the screen.

In general, the video function played an important role in the involvement of the participants. A participant would begin to move a part of his or her body to the music, and the others would see this on the video screen and begin to join in, some using different parts of the body and different gestures. This sometimes evolved into group collaboration where they would simultaneously move their arms and bodies to the beat to create a group affect, and often accompanied by group cries of enjoyment when a particular outcome was achieved. The video controller encouraged participants to work 
Adkins, Barbara, Marie Knox, Steve Dillon, Andrew R. Brown, and Jennifer Summerville. "Digital Technologies and Musical Participation for People with Intellectual Disabilities." New Media and Society 14, no. 7 (2012).

upon their moving images such as using the kaleidoscope effect where they would manipulate their bodies to produce multiple views of themselves, create up-side down views, and images that appeared as though their heads and limbs were joined together. This is illustrated in the following image from a recorded music activity:

Figure 2 Here

Figure 2: Video image of a recorded music piece at a day centre for people with intellectual disabilities

The observation and interview data suggested that the emergent and collaborative relationships supported by the tool promoted a sense of choice and agency in working upon and influencing the form of the music.

Participants responded to their experience using jam2jam in terms of personal and collective enjoyment, for example, 'I love it'. In terms of features they liked the best; they stated that they liked 'the scene' and 'the jazz', the different instruments such as the drums and the guitar, the motions of the sliders, controlling the tempo and 'seeing my face in the background'. The following extract from a group interview following the jam-to-jam activity conveyed the participants' enjoyment of the capacity to explore, discover and appreciate new musical sounds and experiences:

$\mathrm{R}$ : I could hear sound

Interviewer: What sound did you like the best?

$\mathrm{R}: \quad$ Slow, light, fresh

S: I like the guitar one 
Adkins, Barbara, Marie Knox, Steve Dillon, Andrew R. Brown, and Jennifer Summerville. "Digital Technologies and Musical Participation for People with Intellectual Disabilities." New Media and Society 14, no. 7 (2012).

http://nms.sagepub.com/content/early/2012/09/24/1461444812457338.full.

Interviewer: Why do you like the guitar?

S: $\quad$ Because it sounded I liked the sound of it

Interviewer: What did you make it do?

S: $\quad$ Make it go fast and slow

At a number of points in the experiences of the participants with the tool, the recognition of self and others moved into activities premised on relationships enabled by mutual recognition—self confidence, self respect and social esteem. Participants' description of their experiences with the tool sometimes moved to assertions of aesthetic appreciation and preferences in the presence of researchers and fellow participants. One participant suggested that a water sound be added to jam2jam. On several occasions participants at both centres emulated a 'DJ-style' engagement with the sliders, and adopted the performative manner characteristic of this activity in entertainment venues. In the confident adoption of these moves embedded in the jamming activity, participants displayed an expectation of reception and understanding of their music. More generally, the orientation of the tool to the production of a work that could be viewed on video, recorded, and replayed was important in promoting a sense of the creation of a product for others to view and experience. A final video was made that showed the participants smiling, moving to the music and engaging in the activity as group performance rather than individual experimentation. These videos were brought home to show friends and family. In the future, these performances could be conducted in a web-based environment beyond the context of the homes and centres where people with intellectual disability spend their time. 
Adkins, Barbara, Marie Knox, Steve Dillon, Andrew R. Brown, and Jennifer Summerville. "Digital Technologies and Musical Participation for People with Intellectual Disabilities." New Media and Society 14, no. 7 (2012).

http://nms.sagepub.com/content/early/2012/09/24/1461444812457338.full.

\section{Conclusion: the potential of digital tools in creating zones of cultural participation for people with intellectual disability}

For people with intellectual disabilities, the move to deinstitutionalised care has provided new programmes that demarcate and frame the forms of participation they experience. In this context, specific versions of musical practice tend to be privileged due to very limited funding and resources for these programs.

Thus our research problem focused on the nature of relationships of access that might be offered by digital tools, promoting music improvisation as an activity that invites aesthetic agency in music making, and the capacity of participants to assert their own versions of normality and ability in this context.

Studying these relationships required an understanding of the way people with intellectual disabilities are affected by questions of disability and power following deinstitutionalisation. Research on technology and disability had cautioned against assumptions of normalisation. Studies of issues of access and participation for people with intellectual disabilities have recommended a focus on alternative zones of inclusion where people can assert their own versions of ability and normality. Our research brought these principles together, applying them to the question of aesthetic agency, musical practice and the context of the field of culture. As such its contribution involved a conceptualisation of the relationships at stake in these principles, and an exploratory empirical study of what they reveal about these relationships. The hermeneutic approach in Paul Ricoeur's analysis of recognition, applied to processes of aesthetic agency prioritised the potential and possibility of embodied experiences for people with intellectual disabilities 
Adkins, Barbara, Marie Knox, Steve Dillon, Andrew R. Brown, and Jennifer Summerville. "Digital Technologies and Musical Participation for People with Intellectual Disabilities." New Media and Society 14, no. 7 (2012).

http://nms.sagepub.com/content/early/2012/09/24/1461444812457338.full.

(Hughes, 2007), that could enable them to assert their own notions of normality and agency in the context of music improvisation.

Viewed through this conceptual lens our study identified a number of properties of the experience that enabled the exercise of this agency:

- The appeal of a new and different improvisational experience enabled by the jam-to-jam tool, which represented a rich additional resource for creative activity in the day centres.

- The dispositions and qualities of the participants: they undoubtedly enjoyed the music activities programmed for them at the centres which encouraged engagement in music activities. However, the experience with the digital jamming tool introduced a new requirement for improvisation and a less prescribed approach to performance. Their enthusiasm for experimentation, their commitment to understanding and engaging in the activity as group collaboration, and the spontaneity and enjoyment of the work were critical in the formation of the technical and cultural relationships required for the activity.

- The nature of the music itself: while the participants enjoyed and benefited from engagement with known songs during their regular centre based activities, these entailed a more structured and prescribed approach to music making. The nature of the music supported through the digital tool was more reflective of musical forms that embrace difference in form and tonality.

- The integration of video capture in the experience and the capacity to manipulate visual effects were a crucial resource in recognition relationships and aesthetic agency 
Adkins, Barbara, Marie Knox, Steve Dillon, Andrew R. Brown, and Jennifer Summerville. "Digital Technologies and Musical Participation for People with Intellectual Disabilities." New Media and Society 14, no. 7 (2012).

http://nms.sagepub.com/content/early/2012/09/24/1461444812457338.full.

- The commitment of the jam2jam music and technical team to facilitating and encouraging participants' experimentation and engagement.

- The digital tool: jam2jam consisted of an assemblage of interface, controllers and the code and algorithms that enabled the production of the music performance. The combination of software and hardware accomplished what Thrift and French (2002) describe as a bringing or 'holding together' of musical dispositions and competences and the actualisation of this in musical works. A key factor here was the specific space-time context of practice the tool promoted. It enabled the development of a sense of agency and connection between movement of the controllers and the production of different sounds and effects, the connection between sound and vision, including visual effects, which actualised participants' sense of agency. These connections could be explored repeatedly and over time until participants were familiar with these processes. These properties also provided for the experience of improvisation, collaboration and emergence (Sawyer, 2006).

As an exploratory study designed to accompany the development of jam2jam, this study has served to develop conceptual tools and document patterns that now require further focus. In particular, it raises questions of the human-technology inter-relations that potentially deliver the possibilities for music agency and access for people with intellectual disabilities that were described in our study. Moser has suggested that in order to intervene in 'default' assumptions concerning disability, technology and normalisation, our research must employ frameworks that open up questions of the distribution 
Adkins, Barbara, Marie Knox, Steve Dillon, Andrew R. Brown, and Jennifer Summerville. "Digital Technologies and Musical Participation for People with Intellectual Disabilities." New Media and Society 14, no. 7 (2012).

http://nms.sagepub.com/content/early/2012/09/24/1461444812457338.full.

of agency (Moser, 2000). Agency is thus the outcome of relationships between humans, technologies and other entities. This resonates with De la Fuentes' recent review of arts sociology where he recommends an emphasis on the materiality of arts practice and aesthetic experience in understanding aesthetic agency (De la Fuentes, 2010). These are key concepts that could be brought together and operationalised in future studies to capture the details of this agency.

There is a lot at stake in developing a research approach and methodology that provides insight into the ways in which new media technologies figure in aesthetic agency for people with intellectual disabilities. As Finlay et al's (2007) conversation analytic study has shown, the experience of 'not being noticed' inhabits the turn-by-turn process in spoken interaction for this group where gestures, sounds and other phenomena are passed over as turns at talk. Studying the process through which tools can assist to translate these communications into meaningful works requires specifically customised methodological processes. Research strategies are needed that can capture the multiple modalities (Zbikowski, 2009) - musical, linguistic and gestural - through which communication occurs in these contexts. The investigations need to remain open to - and document - the pathways and contexts through which human-technology interfaces support musical agency in line with the preferences, aspirations and experiences of people with intellectual disabilities. 


\section{Acknowledgements}

This work was supported by ACID (the Australasian CRC for Interaction

Design) established and supported under the Cooperative Research Centres

Program through the Australian Government's Department of Education,

Science and Training, and the Endeavour Foundation, a key non-government disability service provider in Australia.

\section{References}

Booth T (1996) 'Sounds of still voices: issues in the use of narrative methods with people who have learning difficulties'. In: L Barton, (ed) Disability and society: emerging issues and insights. London: Longman, 237-255.

Bourdieu P (1984) Distinction: A social Critique of the judgment of taste. (R. Nice, Trans.) Cambridge, MA: Harvard University Press.

Brown A R, S Dillon, T Kerr and A Sorensen (2009). 'Evolving interactions: agile design for networked media performance'. In: K Jesper, J Paay, S.Viller and R Schultz (eds) OzCHI, Melbourne, Australia. 41-48: CHISIG. 
Adkins, Barbara, Marie Knox, Steve Dillon, Andrew R. Brown, and Jennifer Summerville. "Digital Technologies and Musical Participation for People with Intellectual Disabilities." New Media and Society 14, no. 7 (2012).

Dalton A and K McVilly (2004) 'Ethics guidelines for international multi-centre research involving people with intellectual disabilities' [Guidelines commissioned by the International Association for the Scientific Study of Intellectual Disability (IASSID)]. Journal of Policy \& Practice in Intellectual Disability, 1: 57-70.

De la Fuente E (2007) 'The "new sociology of art”: Putting art back into social science approaches to the arts'. Cultural Sociology, 1 (3): 409-425.

De la Fuente E (2010) 'The artwork made me do it: Introduction to the new sociology of art'. Thesis Eleven, 103 (1): 3-9.

DeNora T (2003) After Adorno: Rethinking Music Sociology. Cambridge: Cambridge University Press.

Dillon, S (2006) ‘jam2jam: networked improvisational musical envrionments’. In: M. Moore and B W Leung (eds) School Music and Teacher Education: a global perspective in the new century. Hong Kong: ISME, 19-30.

Duffy B and Fuller R (2000) ‘Role of Music Therapy in Social Skills Development in Children with Moderate Intellectual Disability'. Journal of Applied Research in Intellectual Disabilities 13 (2): 77-89. 
Adkins, Barbara, Marie Knox, Steve Dillon, Andrew R. Brown, and Jennifer Summerville. "Digital Technologies and Musical Participation for People with Intellectual Disabilities." New Media and Society 14, no. 7 (2012).

http://nms.sagepub.com/content/early/2012/09/24/1461444812457338.full.

Edgerton $\mathrm{Cl}$ (1994) 'The effect of improvisational music therapy on the communicative behaviors of autistic children'. Journal of Music Therapy, 31 (1): 31 62

Finlay W M L, C Antaki and C Walton (2007) ‘On not being noticed: Intellectual disabilities and the non-vocal register'. Intellectual and Developmental Disabilities, 45 (4): $227-245$.

Goggin G and C Newell (2003) Digital disability: the social construction of disability in New Media. Lanham and Oxford: Rowan \& Littlefield.

Hall E (2004) 'Social geographies of learning disability: narratives of exclusion and inclusion'. Area, 36 (3): 298-306.

Hall E and A Kearns (2001) 'Making space for the "intellectual” in geographies of disability'. Health and Place, 7: 237-46.

Hamlin A and P Oakes (2008) 'Reflections on deinstitutionalization in the United Kingdom'. Journal of Policy and Practice in Intellectual Disabilities, 5(1): 47-55.

Hendricks, D R and P Wehman (2009) 'Transition from school to adulthood for youth with Autism Spectrum Disorders: review and recommendations'. Focus on Autism and Other Developmental Disabilities, 24 (2): 77-88. 
Adkins, Barbara, Marie Knox, Steve Dillon, Andrew R. Brown, and Jennifer Summerville. "Digital Technologies and Musical Participation for People with Intellectual Disabilities." New Media and Society 14, no. 7 (2012).

Hickey M (2009) 'Can improvisation be "taught”?: A call for free improvisation in our schools'. International Journal of Music Education, 27 (4): 285-299.

Hooper J, Wigram T, Carson D \& Lindsay, B (2011) 'The practical implication of comparing how adults with and without intellectual disability respond to music'. British Journal of Learning Disabilities 39 (1): 22-28.

Hooper, J, Wigram T, Carson D, and Lindsay B (2008a) 'A review of the music and intellectual disability literature (1943-2006): Part one_Descriptive and philosophical writing'. Music Therapy Perspectives 26 (2): 66-79.

Hooper J, Wigram T, Carson, D, and Lindsay B (2008 b) 'A review of the music and intellectual disability literature (1943-2006): Part twoExperimental writing'. Music Therapy Perspectives 26(2): 80-96.

Hughes B (2007) 'Being disabled: towards a critical social ontology for disability studies'. Disability \& Society 22 (7): 673-684.

lacono T (2006) 'Ethical challenges and complexities of including people with intellectual disability as participants in research'. Journal of Intellectual and Developmental Disability 31: 173-179. 
Adkins, Barbara, Marie Knox, Steve Dillon, Andrew R. Brown, and Jennifer Summerville. "Digital Technologies and Musical Participation for People with Intellectual Disabilities." New Media and Society 14, no. 7 (2012).

http://nms.sagepub.com/content/early/2012/09/24/1461444812457338.full.

Klotz J (2004) 'Sociocultural study of intellectual disability: moving beyond labelling and social constructionist perspectives'. British Journal of Learning Disabilities, 32(2): 93-104.

McClimens A and Gordon F (2009) 'People with intellectual disabilities as bloggers: What's social capital got to do with it anyway?' Journal of Intellectual Disabilities 13 (1): 19-30.

Mansell J (2006) ‘Deinstitutionalisation and community living: Progress, problems and priorities'. Journal of Intellectual and Developmental Disability 31: $65-76$.

Marmande F and Johnson C (1996) 'The laws of improvisation, or the nuptial destruction of jazz'. Yale French Studies 89:155-159.

Metzel D S (2005) 'Places of social poverty and service dependency of people with intellectual disabilities: a case study in Baltimore, Maryland'. Health \& Place 11: 93105.

Moser I (2006) 'Disability and the promises of technology: technology, subjectivity and embodiment within an order of the normal'. Information, Communication \& Society $9(3)$ : 373-395.

Moser I (2000) 'Against normalisation: Subverting norms of ability and 
Adkins, Barbara, Marie Knox, Steve Dillon, Andrew R. Brown, and Jennifer Summerville. "Digital Technologies and Musical Participation for People with Intellectual Disabilities." New Media and Society 14, no. 7 (2012).

Disability'. Science as Culture 9 (2): 201-240.

Nind M. and Seale J (2009) 'Concepts of access for people with learning difficulties: towards a shared understanding'. Disability \& Society 24(3): 273287.

Philo C and Metzel D S (2005) 'Introduction to theme section on geographies of intellectual disability: "outside the participatory mainstream?"' Health \& Place 11: 7785

Ramcharan P (2010) 'Capability and Inclusion'. In: C Bigby and C Fyffe (eds) More than Community Presence: Social Inclusion for People with Intellectual Disability, Proceedings of the Fourth Annual Roundtable on Intellectual Disability Policy. Melbourne: School of Social Work and Social Policy

Renblad K (2000) 'Persons with intellectual disability, social interaction and video telephony: An interview study'. Technology and Disability, 13: 55-65.

Ricoeur P (2005) The Course of Recognition. Cambridge, Massachusetts; London, England: Harvard University Press

Rubin S, Biklen D, Kasa-Hendrickson C, Kluth P, Cardinal DN and Broderick A (2001) 'Independence, participation, and the meaning of intellectual ability'. Disability \& Society 16 (3): 415-429.

Sawyer K (2006) ‘Group creativity: musical performance and collaboration’. 
Adkins, Barbara, Marie Knox, Steve Dillon, Andrew R. Brown, and Jennifer Summerville. "Digital Technologies and Musical Participation for People with Intellectual Disabilities." New Media and Society 14, no. 7 (2012).

http://nms.sagepub.com/content/early/2012/09/24/1461444812457338.full.

Psychology of Music, 34: 148-165.

Sawyer K (2000) 'Improvisation and the creative process: Dewey,

Collingwood, and the aesthetics of spontaneity'. The Journal of Aesthetics

and Art Criticism 58 (2): 149-161.

Schalock R L (2010) ‘International perspectives on intellectual

Disability' In: K D Keith (ed) Cross-cultural psychology:

Contemporary themes and perspectives. New York: Wiley-Blackwell, 312 328

Schelly D (2008) 'Problems associated with choice and quality of life for an individual with intellectual disability: a personal assistant's reflexive ethnography'. Disability \& Society 23 (7): 719-732.

Scott A (2004) 'Sittin' in: Barry Harris's use of the jam session as a jazz pedagogical device'. Journal of Popular Music Studies 16 (3): 283-290.

Skewes K and Wigram T (2002) 'A Review of Current Practice in Group Music Therapy Improvisations'. British Journal of Music Therapy 16 (1): 46 - 55.

Stephenson J (2006) 'Music Therapy and the Education of Students with Severe Disabilities'. Education and Training in Developmental Disabilities 41 (3): 290-299. 
Adkins, Barbara, Marie Knox, Steve Dillon, Andrew R. Brown, and Jennifer Summerville. "Digital Technologies and Musical Participation for People with Intellectual Disabilities." New Media and Society 14, no. 7 (2012).

http://nms.sagepub.com/content/early/2012/09/24/1461444812457338.full.

Thrift N and French S (2002) 'The Automatic Production of Space'.

Transactions of the Institute of British Geographers, New Series 27 (3): 309335.

Walker P (1999) 'From community presence to sense of place: community experiences of adults with developmental disabilities'. Journal of the Association for Persons with Severe Handicaps 24 (1): 23-32.

Warde A (2004) 'Practice and field: revising Bourdieusian concepts'. CRIC Discussion Paper 65, April 2004.

Zbikowski L (2009) 'Music, language and multimodal metaphor' in C Forceville and E Urios-Aparisi (eds) Multimodal Metaphor. Berlin: Mouton-de Gruyter.

Zorn J (2004) 'The Game Pieces'. In: C Cox and D Warner (eds) Audio

Culture: Readings in Modern Music. Continuum Press: New York

Websites

ACID Network Jamming:

http://www.acid.net.au/index0b83.html?option=com content\&task=view\&id=64\&ltemi $\underline{d=134}$

Jam2jam website: http://explodingart.com/jam2jam/jam2jam/Home/Home.html

Research with participants with disabilities:

http://explodingart.com/jam2jam/jam2jam/Research/Entries/2010/7/25 Endeavour F 
Adkins, Barbara, Marie Knox, Steve Dillon, Andrew R. Brown, and Jennifer Summerville. "Digital Technologies and Musical Participation for People with Intellectual Disabilities." New Media and Society 14, no. 7 (2012).

http://nms.sagepub.com/content/early/2012/09/24/1461444812457338.full.

oundation.html

http://explodingart.com/jam2jam/jam2jam/Home/Entries/2011/11/19 Together Red

$\underline{\text { Record Launch.html }}$

Videos of jam2jam: Jam2jam Documentary: http://youtu.be/1wjy534u3h0

Jam2jam Video site: http://www.youtube.com/user/jam2jamVideo 
Adkins, Barbara, Marie Knox, Steve Dillon, Andrew R. Brown, and Jennifer Summerville. "Digital Technologies and Musical Participation for People with Intellectual Disabilities." New Media and Society 14, no. 7 (2012).

http://nms.sagepub.com/content/early/2012/09/24/1461444812457338.full.
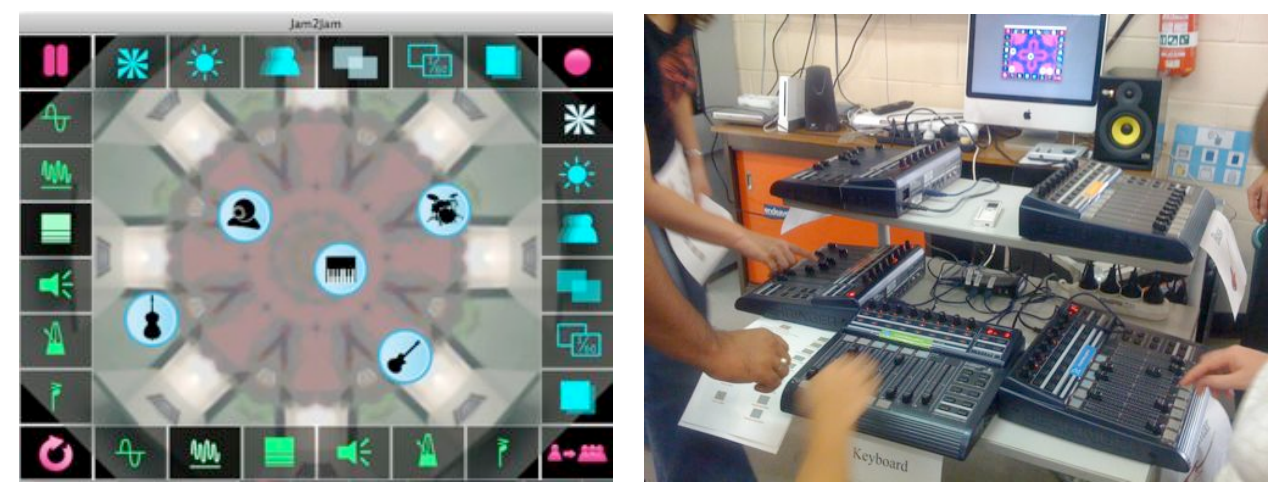

Figure 1 The jam2jam AV Interface showing graphic symbols for parameters and instruments (left), and MIDI-based slider controllers (right). 
Adkins, Barbara, Marie Knox, Steve Dillon, Andrew R. Brown, and Jennifer Summerville. "Digital Technologies and Musical Participation for People with Intellectual Disabilities.” New Media and Society 14, no. 7 (2012).

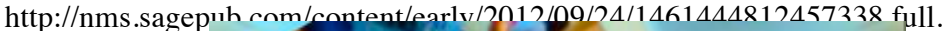

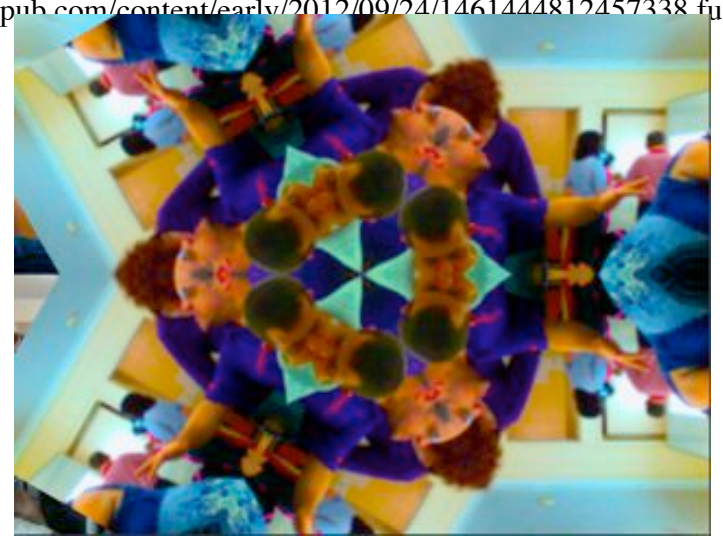

Figure 2 Video image of a recorded music piece at a day centre for people with intellectual disabilities 